\title{
BMJ Open Triage tests for identifying atrial fibrillation in primary care: a diagnostic accuracy study comparing single-lead ECG and modified BP monitors
}

\author{
Karen Kearley, ${ }^{1}$ Mary Selwood, ${ }^{1}$ Ann Van den Bruel, ${ }^{1}$ Matthew Thompson,,${ }^{1,2}$ \\ David Mant, ${ }^{1}$ FD Richard Hobbs, ${ }^{1}$ David Fitzmaurice, ${ }^{3}$ Carl Heneghan ${ }^{1}$
}

To cite: Kearley K, Selwood M, Van den Bruel A, et al. Triage tests for identifying atrial fibrillation in primary care: a diagnostic accuracy study comparing single-lead ECG and modified BP monitors. BMJ Open 2014:4:e004565.

doi:10.1136/bmjopen-2013004565

- Prepublication history and additional material for this paper is available online. To view these files please visit the journal online (http://dx.doi.org/10.1136/ bmjopen-2013-004565).

Received 27 November 2013 Revised 10 March 2014 Accepted 11 March 2014

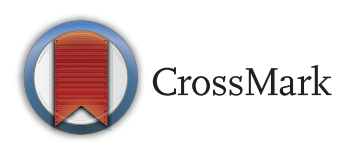

\footnotetext{
${ }^{1}$ Nuffield Department of Primary Care Health Sciences, University of Oxford, Oxford, UK ${ }^{2}$ Department of Family Medicine, University of Washington, Seattle, Washington, USA

${ }^{3}$ Primary Care Clinical Sciences, University of Birmingham, Birmingham, UK
}

Correspondence to Dr Matthew Thompson; mjt@uw.edu

\section{ABSTRACT}

Objective: New electronic devices offer an opportunity within routine primary care settings for improving the detection of atrial fibrillation (AF), which is a common cardiac arrhythmia and a modifiable risk factor for stroke. We aimed to assess the performance of a modified blood pressure (BP) monitor and two singlelead ECG devices, as diagnostic triage tests for the detection of $\mathrm{AF}$.

Setting: 6 General Practices in the UK.

Participants: 1000 ambulatory patients aged 75 years and over.

\section{Primary and secondary outcome measures:}

Comparative diagnostic accuracy of modified BP monitor and single-lead ECG devices, compared to reference standard of 12-lead ECG, independently interpreted by cardiologists.

Results: A total of 79 participants $(7.9 \%)$ had AF diagnosed by 12-lead ECG. All three devices had a high sensitivity (93.9-98.7\%) and are useful for ruling out AF. WatchBP is a better triage test than Omron autoanalysis because it is more specific- $89.7 \%$ (95\% $\mathrm{Cl} 87.5 \%$ to $91.6 \%)$ compared to $78.3 \%(95 \% \mathrm{Cl}$ $73.0 \%$ to $82.9 \%$ ), respectively. This would translate into a lower follow-on ECG rate of $17 \%$ to rule in/rule out AF compared to $29.7 \%$ with the Omron text message in the study population. The overall specificity of single-lead ECGs analysed by a cardiologist was $94.6 \%$ for Omron and $90.1 \%$ for Merlin.

Conclusions: WatchBP performs better as a triage test for identifying AF in primary care than the singlelead ECG monitors as it does not require expertise for interpretation and its diagnostic performance is comparable to single-lead ECG analysis by cardiologists. It could be used opportunistically to screen elderly patients for undiagnosed AF at regular intervals and/or during BP measurement.

\section{INTRODUCTION}

Atrial fibrillation (AF) is the most common cardiac arrhythmia; present in more than $10 \%$ of patients aged 75 years or over, ${ }^{12}$ and

\section{Strengths and limitations of this study}

Three devices for detecting atrial fibrillation (AF) were tested on an unselected elderly primary care population of 1000 individuals.

- The prevalence of AF expected for this setting, which allowed us to determine the operating characteristics of each monitor with precision.

- Our population is generalisable to similar primary care settings worldwide.

- A reference standard (12-lead ECG) was performed on all patients, and interpreted blindly by cardiologists.

- The specificity of one cardiologist was substantially lower than the other three.

- Only 12 new cases of AF were detected in this setting, which is as expected for a primary care population such as this.

it significantly increases morbidity and mortality. ${ }^{3}$ The main significance of $\mathrm{AF}$ is as a major independent risk factor for stroke and thromboembolism, ${ }^{4}$ particularly in older patients. A large evidence base supports the efficacy of oral anticoagulation in reducing AF stroke risk by two-thirds. ${ }^{5}$ AF may be asymptomatic but can be identified by detecting a characteristic irregularity in pulse rhythm. Despite this, it was found to be undiagnosed in $3.8 \%$ of patients aged over 75 years in a large UK screening study. Indeed, undiagnosed $\mathrm{AF}$ is associated with $3.8-6.1 \%$ of all strokes. ${ }^{78}$ Given the importance of identifying patients with risk of stroke, the high prevalence of $\mathrm{AF}$ and the effective prevention strategies, there is a strong case to consider screening for $\mathrm{AF}$.

European guidelines for the management of $\mathrm{AF}$ recommended opportunistic pulse assessment by a primary care practitioner with a follow-up ECG for an irregular pulse, an approach which is more cost-effective 
than systematic screening with a 12-lead ECG. ${ }^{6} 9$ In primary care research studies the sensitivity of nurse pulse assessment is high, varying between studies from $87 \%$ to $97 \%$ although it has a lower specificity $(70-81 \%)$ requiring one out of every three or four screened patients to have a follow-up ECG. ${ }^{6} 1011$ This is a significant additional workload and could be a barrier to widespread implementation of opportunistic screening. Although pulse examination is a simple screening technique for $\mathrm{AF}$, its detection relies on a subjective assessment in a busy routine care setting, which may partly explain why AF remains undetected in many patients. Indeed the American Heart Association suggests there is a need to develop strategies to detect AF more effectively in individuals and populations. ${ }^{12}$

Several new electronic devices have the potential to be useful triage tests for $\mathrm{AF}$ and initial studies suggest these have higher specificity than pulse palpation, so could significantly reduce the need for confirmatory 12-lead ECGs-but none have been evaluated in a primary care setting. ${ }^{13-15}$ The National Institute for Health and Care Excellence (NICE) has recently suggested that using a blood pressure (BP) monitor, WatchBP, which is modified to detect irregularity of pulse during BP measurement in the elderly, together with appropriate anticoagulation of those subsequently diagnosed with $\mathrm{AF}$, has the potential to reduce the incidence of stroke. ${ }^{16}$ As stroke is the third leading cause of global disease burden, this would have substantial economic and patient benefits worldwide. ${ }^{16-18}$

\section{METHODS}

\section{Study design}

An observational study comparing the accuracy of a modified BP monitor (WatchBP), and two single-lead ECG devices, one with an autoanalysis function (Omron) and one without (Merlin), with the reference standard of blinded cardiologist diagnosis of AF from a 12-lead ECG.

\section{Participants}

We recruited participants aged 75 years or over, living at home, from six general practices in the UK between May 2011 and October 2012. Having excluded patients with implanted pacemakers or defibrillators, those unable to give informed consent, or patients in whom the general practitioner (GP) considered participation was inappropriate (eg, terminal illness), all other patients over 75 were invited to take part until the sample size had been achieved (ie, 1000 participants had been recruited). A diagnosis of AF recorded in the patient GP record was not used as part of the selection criteria. Consenting study participants identified from GP records and meeting the eligibility criteria attended a $30 \mathrm{~min}$ appointment with a practice nurse for the testing procedure.
Test procedure

All participants received the tests in the same order, which were delivered by nine registered nurses working within the practices.

First, the nurse used WatchBP (Microlife, Switzerland) a modified oscillometric BP monitor which flashes when it detects an irregular pulse during automatic BP measurement.

Second, the nurse then applied an Omron monitor (model HCG-801, Omron Healthcare Europe, the Netherlands) which involved placing one electrode on the bare chest wall $5 \mathrm{~cm}$ below the nipple, while the patient held the other electrode with the right index finger. The monitor records a single-lead ECG tracing, and displays a message indicating the presence of possible AF. The device's analysis algorithm includes several cardiac rhythms which could potentially be AF, including fast and irregular, slow and irregular, irregular and those where analysis is impossible. The single-lead recording and text message were recorded and saved for later downloading and analysis.

Third, the nurse applied a Merlin ECG event recorder (Meditech Ltd, Hungary) which resembles a watch, on participants' left wrist. The participant covered the electrode on the face of the device with the palm of their right hand for $30 \mathrm{~s}$. The recording, with no automated analysis, was saved to a computer for later downloading and analysis. Unlike the Omron, the Merlin monitor does not require removal of any clothing, making it possible for use in public settings, an advantage for participants experiencing an intermittent arrhythmia.

The nurse recorded the results of the WatchBP monitor and the Omron automated text message during the initial examination. Each single-lead ECG trace was sent for interpretation to two independent cardiologists after removing all clinical information and patient identification except for date of birth and the text message (Omron only).

Participants were asked if they had experienced palpitations, racing, pounding, fluttering or irregular heartbeat in the previous 4 weeks.

\section{The reference standard}

A 12-lead ECG was performed on all participants at the end of the visit, using standard procedures. ECGs were independently interpreted by a panel of two cardiologists, blind to all patient identifiers, clinical details, results of triage tests, but not blind to study objectives. They were asked to classify the ECG as AF or atrial flutter (yes/no/do not know). A third cardiologist, also blind to any of the other study results, interpreted the ECG readings for which there was no consensus or if both cardiologists were uncertain about the presence of AF. AF is defined as the absence of distinct 'p' waves, an absolutely irregular RR interval and an atrial cycle length $<200 \mathrm{~ms}(300 \mathrm{bpm})$ on a 12-lead ECG. ${ }^{17}$

To cope with the workload, the task was divided between two separate pairs of cardiologists. All had 
completed cardiology specialist training of between 5 and 6 years. The third cardiologist, whose role was to resolve uncertainty and moderate disagreement, was a specialist electrophysiologist.

\section{Study power and analysis}

We anticipated that the overall prevalence of AF, including both new and prior diagnoses, would be $10 \%$. We therefore estimated that 1000 participants would be needed to provide sufficient precision to estimate the sensitivity of each device with a maximum $95 \%$ CI of $\pm 10 \%$.

We compared the accuracy of the WatchBP, Omron (text and tracing) and Merlin monitors, with a 12-lead ECG for detection of AF. All inconclusive results were treated as positive in our analysis (because in the clinical situation these participants could not have AF ruled out and would need to have a 12-lead ECG). We calculated sensitivity, specificity, likelihood ratios and 95\% CIs for each test. Analyses for both single-lead ECGs were carried out per cardiologist and the results were subsequently combined using a random effects bivariate model calculating overall sensitivity and specificity. ${ }^{19}$ We analysed the interobserver agreement of whether the single-lead ECG trace showed AF between the cardiologists within each pair together with the $\kappa$ value. ${ }^{20}$

We performed subset analyses of the comparative accuracy in patients with and without existing diagnoses of AF (whether or not treated) recorded in GP records. In addition, we modelled a two-stage screening process for AF by examining patients who were WatchBP flash positive and analysing the sensitivity and specificity of the Omron autoanalysis in this subgroup.

Data analysis was performed using Stata V.11S.

\section{RESULTS}

Of 6529 potentially eligible patients, we invited 2673 for a screening visit; no further invitations were sent after the required 1000 sample had been achieved (figure 1). Participants had an average age of 79.7 years (range 75.1-99.8 years), $49.3 \%$ were men (table 1). One hundred and ten had $\mathrm{AF}$ recorded in the medical summary $(11 \%)$, of whom $67(67 / 110,61 \%)$ had evidence of AF on their 12-lead ECG at the study visit. AF was noted in a further 12 participants based on the 12-lead ECG (1.2\%). The three cardiologists could not reach a consensus about whether $\mathrm{AF}$ was present in the 12-lead ECG in one participant, who was therefore excluded from further analyses which are therefore based on 999 participants.

Overall, $50(5 \%)$ participants reported having experienced palpitations, racing, pounding, fluttering or irregular heartbeat during the previous 4 weeks. Of these, 16 were already known, and three were new cases of AF. The interobserver agreement for the 12-lead ECGs was good for both pairs of cardiologists; the $\kappa$ was 0.69 and 0.96 , respectively for pairs 1 and 2 .
Accuracy of WatchBP and Omron autoanalysis

Both WatchBP and Omron autoanalysis were highly sensitive $(94.9 \%$ and $98.7 \%$, respectively; table 2$)$. The specificity of WatchBP was $89.7 \%$ (95\% CI $87.5 \%$ to $91.6 \%$ ) whereas the Omron text message was lower at $76.2 \%$ (95\% CI $73.3 \%$ to $78.9 \%$; figure 2 ).

The higher specificity of WatchBP would translate into a markedly lower follow-on ECG rate of $17 \%$ to rule in/ rule out $\mathrm{AF}$ compared to $29.7 \%$ with the Omron text message. The superior sensitivity of the Omron text message means the false negative rate is lower than with Watch BP $(0.1 \%$ vs $0.4 \%)$.

The prevalence of AF was 1.4\% (11) in the subset of 889 participants who did not have AF recorded on their medical summary. WatchBP and the Omron autoanalysis provided very similar sensitivity and specificity to the total study population and this translated to a follow-on ECG rate of $11 \%$ for WatchBP and 24\% for the Omron text message. Of these follow-on ECGs, $12 \%$ would be true positives for the WatchBP flash compared to $5 \%$ for the Omron text message.

We modelled a two-stage screening process within our total sample, where patients who had a positive result with WatchBP went on to have the Omron autoanalysis. In participants who screened positive using the WatchBP, the sensitivity and specificity of the Omron autoanalysis was $100 \%$ (95\% CI $95.2 \%$ to $100 \%$ ) and $35.8 \%$ (95\% CI $26.2 \%$ to $46.3 \%$ ), respectively. This would slightly reduce the follow-on 12-lead ECG requirement from $17 \%$ to $13.6 \%$.

\section{Accuracy of Omron and Merlin single-lead ECGs interpreted by a cardiologist}

The diagnostic accuracy of the Omron and Merlin single-lead ECGs is shown in figure 3; meta-analysis of the four cardiologist's interpretations gave Omron a summary sensitivity of $94.4 \%$ with a summary specificity of $94.6 \%$; Merlin had a summary sensitivity of $93.9 \%$ with a summary specificity of $90.1 \%$. Cardiologist variability in single-lead ECG analysis is shown in table 3. There was fair interobserver agreement ( $\kappa 0.36$ and 0.28 for Omron and Merlin, respectively) between cardiologist pair 1, and good interobserver agreement ( $\kappa 0.76$ for both devices) between the second pair. The cardiologists were unable to interpret whether the trace showed AF on $0-1.2 \%$ of patients using Omron and $0-7.2 \%$ of occasions using Merlin.

\section{DISCUSSION}

\section{Summary of main results}

Our results show that all the methods that we tested have a high sensitivity for detecting $\mathrm{AF}$ in an elderly primary care population and thus are useful for ruling out AF. During the study we detected 12 new cases of AF and $7.9 \%$ of the study population overall were experiencing $\mathrm{AF}$ at the time of the study consultation. The total prevalence in the study sample was $11 \%$, as some 
Figure 1 Patient recruitment flowchart.

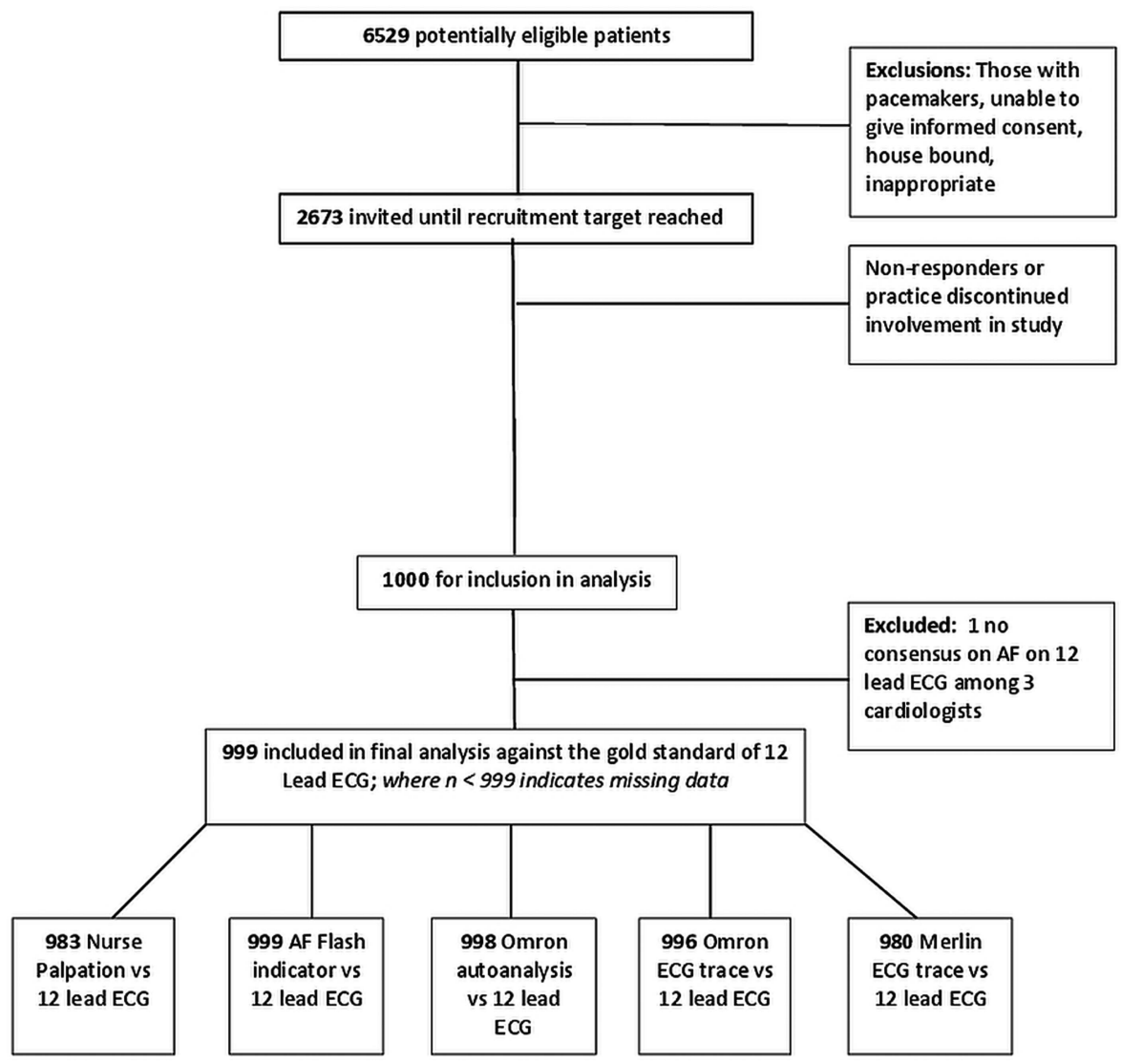

patients have paroxysmal $\mathrm{AF}$ and were not experiencing arrhythmias at the time of the study.

WatchBP outperformed Omron autoanalysis because it is more specific-89.7\% compared to $76.2 \%$ and $78.3 \%$, respectively. The specificity of the single-lead ECGs when interpreted by a cardiologist was comparable with WatchBP. Overall Omron produced fewer inconclusive results and achieved slightly better specificity than Merlin. However cardiologist interpretation of the ECG

\begin{tabular}{ll}
$\begin{array}{l}\text { Table } 1 \text { Characteristics of participants included in } \\
\text { diagnostic accuracy analyses }\end{array}$ \\
\hline Total number of participants & 999 \\
Age (mean) & 79.7 years (range \\
& $75.1-99.8)$ \\
& $(95 \% \mathrm{Cl} 79.4$ to 79.9$)$ \\
Gender (\%, male) & $49.3(95 \% \mathrm{Cl} 46.2$ to \\
& $52.5)$ \\
Comorbidities (from medical summary) \\
AF & $110(11 \%)$ \\
Heart failure & $31(3.1 \%)$ \\
Hypertension & $533(53.3 \%)$ \\
Diabetes & $122(12.2 \%)$ \\
Stroke & $31(3.1 \%)$ \\
Transient ischaemic attack & $65(6.5 \%)$ \\
Participants with AF on & $87(8.7 \%)$ \\
antiarrhythmic medication & \\
\hline AF, atrial fibrillation. & \\
\end{tabular}

trace was a more powerful factor in determining specificity than the type of monitor used. In this study, operator variability in ECG analysis was partly due to differences in expertise, and partly due to trade-offs made by different cardiologists between sensitivity and specificity.

In our elderly population, only $5 \%$ of patients reported experiencing palpitations. Among patients who did report palpitations, prevalence of $\mathrm{AF}$ was $36 \%$. Most of these patients already had a diagnosis of AF.

\section{Strengths and weakness}

The devices were tested on an unselected elderly primary care population. Although our sample did not include the housebound or patients with dementia, we recruited a large population with a prevalence of $\mathrm{AF}$ expected for this setting, which allowed us to determine the operating characteristics of each monitor with precision. We believe our population is generalisable to similar primary care settings worldwide.

The cardiologists analysed ECG recordings in line with recommended practice and all had a minimum of 5 years cardiology specialist training. The specificity of one cardiologist was substantially lower than the other three. Further research with a larger sample of cardiologists would be required to more fully interpret this finding. However, we do not believe this diminishes the reliability of our reference standard since the second pair of blinded cardiologists achieved extremely high 
Table 2 Diagnostic performance of WatchBP and Omron autoanalysis for detecting atrial fibrillation

\begin{tabular}{|c|c|c|}
\hline n=999* Prevalence $7.9 \%$ & WatchBP with AF indicator flash $(95 \% \mathrm{Cl})$ & Omron autoanalysis* $(95 \% \mathrm{Cl})$ \\
\hline Sensitivity (\%) & $94.9(87.5$ to 98.6$)$ & 98.7 (93.2 to100) \\
\hline Specificity (\%) & 89.7 (87.5 to 91.6$)$ & 76.2 (73.3 to 78.9$)$ \\
\hline Positive likelihood ratio & $9.2(7.6$ to 11.2$)$ & 4.15 (3.69 to 4.67$)$ \\
\hline Negative likelihood ratio & $0.057(0.022$ to 0.15$)$ & $0.017(0.0024$ to 0.12$)$ \\
\hline Positive predictive value & 44.1 (36.5 to 51.9$)$ & $26.3(21.3$ to 31.7$)$ \\
\hline Negative predictive value & 99.5 (98.8 to 99.9$)$ & 99.9 (99.2 to 100$)$ \\
\hline
\end{tabular}

*Text message 'irregular' or 'analysis impossible' counted as positive test.

$\mathrm{AF}$, atrial fibrillation.

concordance, and the moderating consultant cardiologist was a specialist electrophysiologist. Having cardiologists rather than GPs ascertain the presence or absence of AF on the Omron or Merlin single-lead ECGs may have risked over estimating accuracy given the likelihood that cardiologists would have greater skill in ECG interpretation. Finally, given that the devices were used sequentially followed by the 12-lead ECG it is theoretically possible that AF may not have been consistently present or absent during the entire procedure, leading to an increase of false positives and false negatives, however we believe this is a small risk given the extremely short-time frame of approximately $10 \mathrm{~min}$.

\section{Comparison with existing literature}

The operating characteristics of WatchBP in this elderly primary care population are comparable to previous studies undertaken in different populations. In a US study of 405 patients attending a cardiology outpatients where $39 \%$ of the sample had an abnormal ECG (AF prevalence $23 \%)$ it had a sensitivity of $95 \% \quad(95 \%$ CI $93 \%$ to $98 \%)$ and specificity of $86 \%$ (95\% CI $84 \%$ to $89 \%$ ) while in a Greek study of a diverse group of 73 individuals (healthy volunteers, outpatients and inpatients) it had similar sensitivity of $93 \%$ (95\% CI $74 \%$ to $99 \%$ ) and specificity of $89 \%$ (95\% CI $79 \%$ to $96 \%)$.

Omron has been evaluated in a German study of 505 patients in a hospital setting where $66 \%$ of the sample had an abnormal ECG (mean age 61 years, $66 \%$ men,
AF prevalence $28 \%$ ) with all analyses (including the reference standard) being undertaken by a single cardiologist. This study reported higher accuracy than we demonstrated, with a sensitivity of $99 \%$ (95\% CI $96 \%$ to $100 \%$ ) and specificity of $96 \%$ (95\% CI $94 \%$ to $98 \%)$ for $\mathrm{AF}$. We are unaware of any AF validation studies for Merlin.

\section{Clinical implications}

Our study supports the recent NICE recommendation that GPs should consider using WatchBP for elderly patients who have a BP measurement in order to opportunistically identify undiagnosed AF. Specificity is comparable with a single-lead ECG analysed by a cardiologist and the result does not require specialist interpretation, which is an advantage over the single-lead ECG monitors and keeps screening straightforward and costs less. It is easily portable for use in housebound patients.

Prior to wider implementation, clinicians need to consider the optimal screening frequency. Assuming a primary care population in which the majority of $\mathrm{AF}$ cases had already been identified, the WatchBP would indicate a 'positive flash' for 1 in 10 patients who would thus require a follow-up 12-lead ECG. As some patients have their BP measured frequently, there is potential for recurrent false positive results and a high demand for 12-lead ECGs. These issues should be addressed to ensure sustainable implementation in primary care.

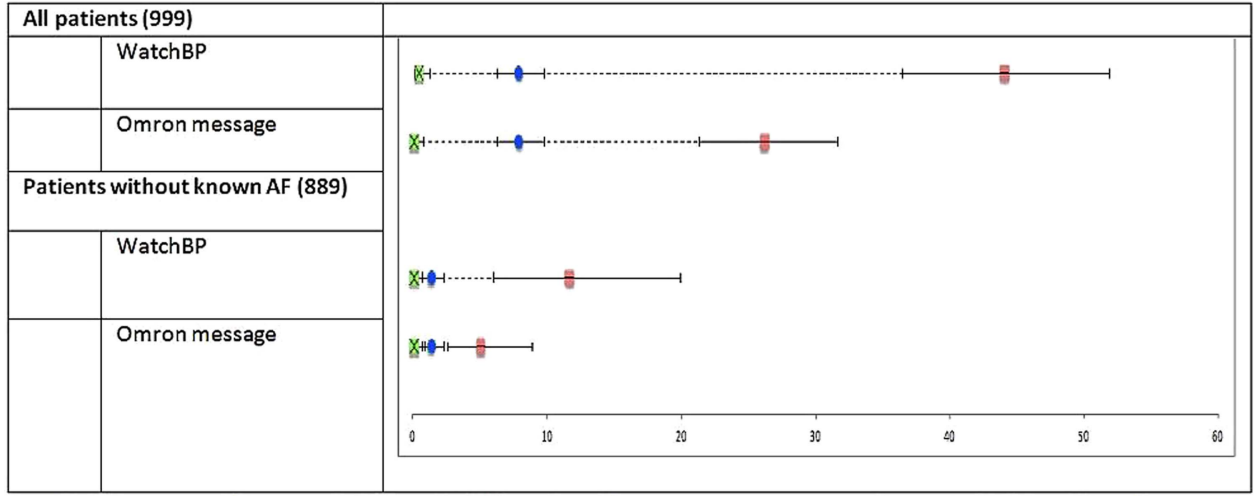

Blue dots = prevalence; red dots: probability of AF after a positive test; green dots: probability of AF after a negative test (each with their $95 \% \mathrm{CI}$ )

Figure 2 Predictive value of WatchBP and Omron autoanalysis for detecting atrial fibrillation (AF). 


\section{OMRON}

\begin{tabular}{|c|c|c|c|c|c|c|c|c|}
\hline Study & TP & FP & FN & TN & Sensitivity $(95 \% \mathrm{Cl})$ & Specificity (95\% Cl) & Sensitivity $(95 \% \mathrm{Cl})$ & Specificity (95\% CI) \\
\hline Cardiologist 1 & 35 & 110 & 2 & 332 & $0.95[0.82,0.99]$ & $0.75[0.71,0.79]$ & $\rightarrow$ & = \\
\hline Cardiologist 2 & 35 & 10 & 2 & 432 & $0.95[0.82,0.99]$ & $0.98[0.96,0.99]$ & $\rightarrow$ & \\
\hline Cardiologist 3 & 38 & 12 & 4 & 463 & $0.90[0.77,0.97]$ & $0.97[0.96,0.99]$ & $\rightarrow$ & \\
\hline Cardiologist 4 & 41 & 25 & 1 & 450 & $0.98[0.87,1.00]$ & $0.95[0.92,0.97]$ & & 문 \\
\hline
\end{tabular}

MERLIN

$\begin{array}{lrrrrr}\text { Study } & \text { TP } & \text { FP } & \text { FN } & \text { TN } & \text { Sensitivity }(95 \% \text { CI) } \\ \text { Cardiologist 1 } & 34 & 105 & 1 & 326 & 0.97[0.85,1.00] \\ \text { Cardiologist 2 } & 32 & 21 & 3 & 410 & 0.91[0.77,0.98] \\ \text { Cardiologist 3 } & 41 & 40 & 1 & 432 & 0.98[0.87,1.00] \\ \text { Cardiologist 4 } & 37 & 41 & 5 & 431 & 0.88[0.74,0.96]\end{array}$

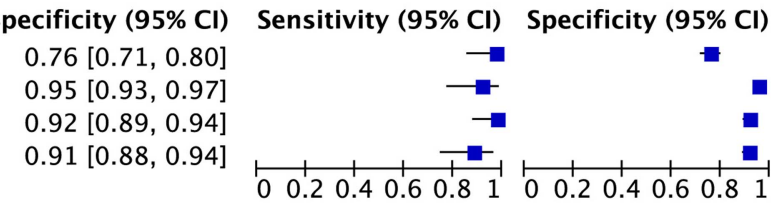

Figure 3 Forest plot of diagnostic accuracy per cardiologist of Omron and Merlin single-lead ECGs.

Paroxysmal AF presents a particular diagnostic challenge because patients may not be experiencing an arrhythmia when they are being screened with one-off measures. The potential for patients to undertake selfmonitoring and to capture an ECG tracing when an arrhythmia is occurring are desirable features of an AF screening method. In our study, only $5 \%$ of patients reported experiencing palpitations, which would limit the usefulness of asking patients to record a single-lead ECG at the time of symptoms. Among the patients who did report palpitations, the prevalence of AF was $36 \%$, but most of these patients already had a diagnosis of AF which might have improved their awareness of an arrhythmia.
WatchBP will not identify atrial flutter, which is also a risk factor for stroke. The prevalence of this is much lower than AF; in our study it was $0.4 \%$.

\section{Future research}

Screening for AF is not yet routine practice in primary care, therefore determining not only the optimal device, but also the most appropriate and cost-effective process is a priority. This could include an impact analysis using WatchBP as a triage test to evaluate its effectiveness (and cost-effectiveness) for improving the detection of AF and prevention of stroke. Determining the cost-effectiveness of these devices would also have to include doctor time for interpretation of ECGs.

\begin{tabular}{|c|c|c|c|}
\hline n=999 Prevalence $7.9 \%$ & Interpretation & Omron single-lead ECG (95\% Cl) & Merlin single-lead ECG $(95 \% \mathrm{Cl})$ \\
\hline \multirow[t]{4}{*}{ Sensitivity (\%) } & Cardiologist: 1 & 95 (82 to 99$)$ & 97 (85 to 100$)$ \\
\hline & 2 & 95 (82 to 99$)$ & 91 (77 to 98$)$ \\
\hline & 3 & 90 (77 to 97$)$ & $98(87$ to 100$)$ \\
\hline & 4 & 98 (87 to 100$)$ & 88 (74 to 96$)$ \\
\hline \multirow[t]{4}{*}{ Specificity (\%) } & Cardiologist: 1 & 75 (71 to 79$)$ & 76 (71 to 80$)$ \\
\hline & 2 & 98 (96 to 99 ) & 95 (93 to 97 ) \\
\hline & 3 & 97 (96 to 99 ) & 92 (89 to 94$)$ \\
\hline & 4 & 95 (92 to 97$)$ & 91 (88 to 94$)$ \\
\hline \multirow[t]{4}{*}{ Positive likelihood ratio } & Cardiologist: 1 & $3.8(3.2$ to 4.6$)$ & 3.99 (3.3 to 4.8$)$ \\
\hline & 2 & $41.8(22.5$ to 77.5$)$ & $18.8(12.2$ to 28.8$)$ \\
\hline & 3 & 35.8 (20.3 to 63.1$)$ & 11.5 (8.5 to 15.6$)$ \\
\hline & 4 & 18.5 (12.6 to 27.2$)$ & 10.1 (7.4 to 13.9$)$ \\
\hline \multirow[t]{4}{*}{ Negative likelihood ratio } & Cardiologist: 1 & 0.07 (0.02 to 0.28$)$ & $0.04(0.01$ to 0.26$)$ \\
\hline & 2 & $0.05(0.01$ to 0.21$)$ & 0.09 (0.03 to 0.27$)$ \\
\hline & 3 & $0.098(0.04$ to 0.25$)$ & $0.03(0.004$ to 0.18$)$ \\
\hline & 4 & $0.02(0.004$ to 0.17$)$ & $0.13(0.06$ to 0.3$)$ \\
\hline \multirow[t]{4}{*}{ Positive predictive value } & Cardiologist: 1 & 24.1 (17.4 to 31.9$)$ & 24.5 (17.6 to 32.5$)$ \\
\hline & 2 & 77.8 (62.9 to 88.8$)$ & 60.4 (46.0 to 73.5$)$ \\
\hline & 3 & 76.0 (61.8 to 86.9$)$ & 50.6 (39.3 to 61.9$)$ \\
\hline & 4 & 62.1 (49.3 to 73.8 ) & 47.4 (36.0 to 59.1$)$ \\
\hline \multirow[t]{4}{*}{ Negative predictive value } & Cardiologist: 1 & 99.4 (97.9 to 99.9$)$ & 99.7 (98.3 to 100$)$ \\
\hline & 2 & 99.5 (98.3 to 99.9$)$ & 99.3 (97.9 to 99.8$)$ \\
\hline & 3 & 99.1 (97.8 to 99.8$)$ & 99.8 (98.7 to 100$)$ \\
\hline & 4 & 99.8 (98.8 to 100$)$ & 98.9 (97.3 to 99.6$)$ \\
\hline
\end{tabular}




\section{CONCLUSION}

WatchBP performs better than the single-lead ECG monitors as a triage test for identifying AF in primary care as it does not require any expertise for interpretation and their diagnostic performance is comparable. It could be used to opportunistically screen elderly patients for undiagnosed AF at regular intervals and/or during BP measurement. It has advantages over pulse assessment because the result is objective and it also has a higher specificity, keeping follow-up 12-lead ECGs to a minimum.

Contributors KK, MS, DM, FDRH, DF and $\mathrm{CH}$ initiated the study and designed the protocol. $\mathrm{KK}, \mathrm{MS}, \mathrm{AVdB}, \mathrm{MT}$ and $\mathrm{CH}$ were involved in the conduct and analysis of the trial. MS oversaw data management and monitoring of protocol adherence throughout the trial. KK, AVdB and MT cowrote the manuscript. MS, DM, CH, FDRH and DF contributed to writing the manuscript.

Funding This publication presents independent research funded by the National Institute for Health Research (NIHR) under its Programme Grants for Applied Research funding scheme (RP-PG-0407-10347) and the NIHR School for Primary Care Research.

Competing interests We received subsidised monitors from Microlife, Omron and Merlin for the submitted work; FDRH has received free or subsidised BP equipment from Omron and Microlife for other research.

Patient consent Obtained.

Ethics approval Ethical approval for the study was granted by NRES Committee South Central-Oxford A.

Provenance and peer review Not commissioned; externally peer reviewed.

Data sharing statement Data on comparative accuracy are available from authors on request, sending email to the corresponding author.

Open Access This is an Open Access article distributed in accordance with the Creative Commons Attribution Non Commercial (CC BY-NC 3.0) license, which permits others to distribute, remix, adapt, build upon this work noncommercially, and license their derivative works on different terms, provided the original work is properly cited and the use is non-commercial. See: http:// creativecommons.org/licenses/by-nc/3.0/

\section{REFERENCES}

1. NICE. Atrial fibrillation: national clinical guideline for management in primary and secondary care. Royal College of Physicians, 2006.

2. Fitzmaurice DA, Hobbs FDR, Jowett S, et al. Screening versus routine practice for detection of atrial fibrillation in people aged 65 or over: cluster randomised controlled trial. BMJ 2007;335: 383-6.
3. Miyasaka $\mathrm{Y}$, Barnes ME, Bailey $\mathrm{KR}$, et al. Mortality trends in patients diagnosed with first atrial fibrillation - a 21-year community study. J Am Coll Cardiol 2007;49:986-92.

4. Wolf PA, Abbott RD, Kannell WB. Atrial fibrillation as an independent risk factor for stroke: the Framingham study. Stroke 1991;22: 983-8.

5. Aguilar MI, Hart R. Oral anticoagulants for preventing stroke in patients with non-valvular atrial fibrillation and no previous history of stroke or transient ischemic attacks. Cochrane Database Syst Rev 2005;(3):CD001927.

6. Hobbs F, Fitzmaurice DA, Mant $\mathrm{J}$, et al. A randomised controlled trial and cost-effectiveness study of systematic screening (targeted and total population screening) versus routine practice for the detection of atrial fibrillation in people aged 65 and over. The SAFE study. Health Technol Assess 2005;9:1-74.

7. Lin HJ, Wolf PA, Benjamin EJ, et al. Newly diagnosed atrial fibrillation and acute stroke. The Framingham Study. Stroke 1995;26:152730.

8. Liao J, Khalid Z, Scallan C, et al. Noninvasive cardiac monitoring for detecting paroxysmal atrial fibrillation or flutter after acute ischemic stroke: a systematic review. Stroke 2007;38:2935-40.

9. Camm AJ, Lip GY, De Caterina R, et al.; ESC Committee for Practice Guidelines (CPG). 2012 focused update of the ESC Guidelines for the management of atrial fibrillation. An update of the 2010 ESC Guidelines for the management of atrial fibrillation. Eur Heart J 2012;33:2719-47.

10. Sudlow M, Rodgers $\mathrm{H}$, Kenny RA, et al. Identification of patients with atrial fibrillation in general practice: a study of screening methods. BMJ 1998;317:327.

11. Morgan S, Mant D. Randomised trial of two approaches to screening for atrial fibrillation in UK general practice. $\mathrm{Br} J$ Gen Pract 2002;52:373-80.

12. Estes N, Sacco R, Al-Khatib S, et al. American Heart Association Atrial Fibrillation Research Summit. Circulation 2011;124:363-72.

13. Wiesel J, Fitzig L, Herschman Y, et al. Detection of atrial fibrillation using a modified microlife blood pressure monitor. Am J Hypertens 2009;22:848-52.

14. Stergiou G, Karpettas N, Protogerou A, et al. Diagnostic accuracy of a home blood pressure monitor to detect atrial fibrillation. J Hum Hypertens 2009;23:654-65.

15. Kaleschke G, Hoffman B, Drewitz I, et al. Prospective, multicentre validation of a simple, patient-operated electrocardiographic system for the detection of arrhythmias and electrocardiographic changes. Europace 2009;11:1362-8.

16. NICE. WatchBP Home A for opportunistically detecting atrial fibrillation during diagnosis and monitoring of hypertension. 2013

17. Lozano R, Naghavi M, Foreman K, et al. Global and regional mortality from 235 causes of death for 20 age groups in 1990 and 2010: a systematic analysis for the Global Burden of Disease Study 2010. Lancet 2012;380:2095

18. Harris K, Edwards D, Mant J. How can we best detect atria fibrillation? J R Coll Physicians Edinb 2012;42(Suppl 18): 5-22.

19. Macaskill P, Gatsonis C, Deeks J, et al. Chapter 10: analysing and presenting results. In: Deeks JJ, Bossuyt PM, Gatsonis C, eds. Cochrane handbook for systematic reviews of diagnostic test accuracy version 1.0. The Cochrane Collaboration, 2010:1-61.

20. Altman D. Practical statistics for medical research. Chapman and Hall/CRC, 1991 